\title{
Measurement Device
}

National Cancer Institute

\section{Source}

National Cancer Institute. Measurement Device. NCI Thesaurus. Code C81182.

Any device used to determine the dimension of an object. 